\title{
Antibiotic Resistance in Blood Culture Samples from Patients Referred to Razi Laboratory of Rasht, 2006-2011
}

\author{
Fatemeh Saeidynia1, Faeze Keihanian ${ }^{2 *}$, Amin Saeidynia ${ }^{2}$ \\ ${ }^{1}$ Guilan University of Medical Sciences, Rasht, Iran \\ ${ }^{2}$ Scientific \& Research Club of Student Basij, Guilan University of Medical Sciences, Rasht, Iran \\ Email: a.saeidynia@yahoo.com, ${ }^{*}$ Faezekeihanian@yahoo.com
}

Received 29 June 2014; revised 29 July 2014; accepted 18 August 2014

Copyright (C) 2014 by authors and Scientific Research Publishing Inc.

This work is licensed under the Creative Commons Attribution International License (CC BY). http://creativecommons.org/licenses/by/4.0/

(c) (i) Open Access

\section{Abstract}

Introduction: Irregular wide antibiotic administration by physicians and over the counter use of them had led to inappropriate or non-essential choice of antibiotic. If blood related diseases like sepsis and bacteremia, have not been diagnosed and treated as soon as possible, they can make high complications and mortalities. This study was conducted to survey of epidemiological changes about frequency of micro-organisms in blood cultures in Razi laboratory of Rasht during 2006 to 2011. Material \& Methods: This is a descriptive retrospective study performed by information database of Razi laboratory. For all of positive samples addressed for blood culture, anti-biograms had been done by method of Kirby \& Bauer and agar diffusion. Last, formation or deformation of growth zone was evaluated and on the basis of standard table, results were classified to three categories of sensitive, intermediate and resistance. All of common standard antibiotic disks had been used in this survey. Results: In all cases which addressed for culture (466 cases) 43 subjects $(9.22 \%)$ were positive and one of them was contaminated. The most frequent bacteria separated from the blood cultures were respectively: coagulase negative Staphylococcus, Escherichia coli, Pseudomonas aeruginosa, Klebsiella and coagulase positive Staphylococcus. Relation between type of bacteria and gender of patients was significant $(P=0.001)$ in which in female cases it was the most frequent. The most resistance in all of applied antibiotics in coagulase negative Staphylococcus group was $60.2 \%$, in gram negative non non-fermentative bacillus group was $43.16 \%$ and in gram negative intestinal bacteria group was $39.25 \%$. Discussion \& Conclusion: Although there are similar results in the pattern of resistance in comparison of other studies, pattern of this study is unique and exclussive for Rasht city and it is based on hygienic condition of Guilan province. Because of being respective and variables were not considerd by opinions of researchers of this study, it is necessary to conduct a prospective and continous study in this wide level.

\footnotetext{
${ }^{*}$ Corresponding author.

How to cite this paper: Saeidynia, F., Keihanian, F. and Saeidynia, A. (2014) Antibiotic Resistance in Blood Culture Samples from Patients Referred to Razi Laboratory of Rasht, 2006-2011. Advances in Infectious Diseases, 4, 165-172. http://dx.doi.org/10.4236/aid.2014.43023
} 


\section{Keywords}

\section{Pattern of Resistance, Antibiotic, Rasht, North of Iran}

\section{Introduction}

Antibiotic therapy is the use of chemical agents against micro organisms that affect on death or inhibition of their growth [1]. Antibiotics are used for more than fifty years to treat infections quickly and effectively and till now, many changes in the types of consumable antibiotics, sensitive and bacteria resistance are happening. It could be due to some reasons, including long-term use of antibiotics [2] the rise of emerging and reemerging infectious diseases, empirical use of antibiotics by patients over the counter (access to the antibiotics without a prescription) [3] or indiscriminate prescribing of antibiotics by doctors which leads to the selection of inappropriate or unnecessary antibiotics. Increasing bacterial resistance to antibiotics, in addition to treatment failure, imposes an economic burden to patients [4]. Loss of appropriate treatment and rapid diagnosis in blood disorders such as sepsis and bacteremia, will cause high mortality and morbidity. Blood culture reports positive in $30 \%-60 \%$ of cases in which gram-negative bacteria, gram positive and fungi are respectively the most common causes. In these cases it must be made at least two blood cultures. However, because of the urgency in blood disorders and requirement to immediate action, should not be kept waiting for an answer and culture and the empiric treatment should immediately begin [5] [6]. Bacteremia and dissemination to different parts of the microorganisms can cause organ dysfunction and patient mortality [7] [8]. Annually worldwide, about 200,000 cases of bacteremia occur, and about 20 and 50 percent of them lead to death [9] [10]. Due to the growing trend of increased number of bacteria resistant to treatment, because of lack of knowledge about the side effects of unnecessary use of antibiotics, the need for study of bacterial resistance to antibiotics used in any clinical laboratory antibiogram test, regularly, seems to be required [11]. According to Razi laboratory of Rasht city, which is one of the accredited laboratories and reference, this study was performed to epidemiological changes in the abundance of microorganisms and their antibiogram sensitivity changes in blood cultures during 2006 to 2011. Awareness of the resistance and sensitivity in identifying and using appropriate empiric therapy can be effective.

\section{Materials and Methods}

This was a retrospective study which performed by using data recorded in the computer and register system of Razi laboratory on blood culture.

In the sample of patients who had blood culture, blood culture bottles were sent to the laboratory and it was kept for 7 weeks in 37 degree in incubator and usually three blood cultures is taken from each patient. Of course sometimes we had avail or two of blood culture vials. Maintained during after the first 24 hours, a sub-culture sample was done on Mackanky and blood agar settings and after negative culture result, second sub-culture sample was taken after 72 hours of arrival and again after negative result of cultures after 10 days, final subculture has done. In each of the above steps, the result of positive cultures were obtained from colonies then diagnostic and biochemical tests were taken, finally the type of bacteria determined.

In this study, all the samples that were sent for blood culture. All positive blood cultures in the laboratory were examined and all it took antibiotic disc method agar diffusion method of Kirby \& Bauer. First, a suspension of isolated colonies standard McFarland preparation and a sterile swab along the flame from bacterial suspensions and withdrawals and the level of Muller Hinton culture medium agar culture dense was done on the surface of plate then antibiotic discs with sterile forceps, placed on the plates. After $24 \mathrm{~h}$ incubation at $37^{\circ} \mathrm{C}$, the formation or non-formation of growth zone investigated based on the results of a standard table which is classified in three forms: susceptible, resistant or intermediate. Antibiotics used in this study consisted of: Amikacin, Amoxicillin, Carbenicillin, Cephalothin, Chloramphenicol, Ciprofloxacin, Cefoxitin, Sulfamethoxazole-Trimethoprim, Erythromycin, Gentamicin, Kanamycin, Nalidixic acid, Oxacillin, Penicillin, Rifampin, Tetracycline, Tobramycin, Vancomycin, Imipenem, Cefotaxime, Clindamycin, Cefixime, Ofloxacin, Piperacillin, Ceftazidime, Cefuroxime, Cefepime, Ticarcillin, Ceftriaxone, Streptomycin, Azithromycin, Norfloxacin. 


\section{Results}

The entire sample was sent for blood culture (466 samples) 43 of them were positive (22.9\%), and one sample was contaminated. $44.8 \%$ of Positive cases were male and $55.2 \%$ of positive cases, were female. The frequency of bacteria isolated from blood cultures of patients are shown in Table 1.

The prevalence of bacteria isolated from blood cultures taken were: coagulase-negative staphylococci, Escherichia Coli, Pseudomonas aeruginosa, Klebsiella, coagulase-positive Staphylococcus aureus, Bacillus cereus, Pneumococci, enterococci, P. aeruginosa, Shigella, Acinetobacter. Three major groups of blood cultures consisted of coagulase negative staphylococci (46.5\%), entericgram-negative bacteria (25.6\%) and gram-negative bacilli Non-fermentative (11.6\%). These groups included a total of $83.7 \%$ of positive cases. Significant relationship between gender and the prevalence of bacteria was found $(P=0.001)$ so that the amount was more common in females. The most common antibiotics used in the total samples are shown in Table 2.

As considered in the table, three commonly used antibiotics areciprofloxacin, sulfamethoxazole-trimethoprim and tetracycline. The test results antibiotic resistance determination to common bacteria in blood cultures is shown in Table 3.

The results of this study showed that 20 antibiotics used to front the highest strength among the groups was reported: $60.2 \%$ of the total resistance to antibiotics in group 3, 43.16\% of the total resistance to antibiotics in Group 1 and 39.25\% of total antibiotic resistance was observed in Group 2.

Sensitivity and resistance to used antibiotics are shown in Table 4. As it is seen in the table, most sensitive antibiotics are in order of: Chloramphenicol, Vancomycin and Ciprofloxacin. Most resistance is also related to: Cefixime, Amoxicillin and Cefoxitin.

\section{Discussion \& Conclusion}

Because of increasing deaths of diseases associated with blood culture, such as bacteremia, septicemia and so on and increasing antibiotic resistance, doing this research in this area is essential. So the results of blood cultures in suspected patients have an important role demographically. In the study, we take Razi laboratory as a reference laboratory Rasht is contrary to other studies, in all cases with positive blood culture contamination was detected in all cases except for one case, all positive samples infected respectively [12]).

Common bacteria in this study were similar to those in other studies. In Momeishi and colleagues’ study 2005, the most common bacteria in blood cultures, respectively, were: coagulase negative staphylococci, Staphylococcus aureus, Klebsiella, E. coli and Pseudomonas aeruginosa [13]. In the present study, coagulase negative Staphylococcus, E. coli, Pseudomonas, Klebsiella and Staphylococcus coagulase positive, respectively, the most common microorganisms in blood cultures were positive. Sedighian and colleagues in a study that was conducted in 2009 in Babol the most common microorganisms in 115 blood culture-positive samples, include: Coagulase negative Staphylococcus (25.6\%), E. coli (4.7\%), Pseudomonas (1.5\%) and Klebsiella (1.5\%) [11].

Table 1. Frequency of bacteria isolated from blood cultures of patients.

\begin{tabular}{cc}
\hline Bacteria isolated from the blood cultures & Percent \\
\hline Coagulase-negative staphylococci & 46.5 \\
Escherichia coli & 16.3 \\
Pseudomonas aeruginosa & 9.3 \\
Klebsiella & 7 \\
Coagulase-positive staphylococci & 4.7 \\
Bacilluscereus & 4.7 \\
Pneumococci & 4.7 \\
Enterobacter aeruginosa & 2.3 \\
Shigella & 2.3 \\
Acinetobacter & 2.3 \\
\hline
\end{tabular}


Table 2. Frequency of antibiotics used in antibiogram of blood culture samples taken.

\begin{tabular}{|c|c|c|}
\hline Tier & Antibiotics & Percentage use of antibiogram \\
\hline 1 & Ciprofloxacin & 95.3 \\
\hline 2 & Sulfamethoxazole-trimethoprim & 81.39 \\
\hline 3 & Tetracycline & 60.4 \\
\hline 4 & Penicillin & 53.4 \\
\hline 5 & Erythromycin & 48.8 \\
\hline 6 & Vancomycin & 46.5 \\
\hline 7 & Amikacin & 44.1 \\
\hline 8 & Ceftriaxone & 41.8 \\
\hline 9 & Imipenem & 39.5 \\
\hline 10 & Clindamycin & 39.5 \\
\hline 11 & Ticarcillin & 39.5 \\
\hline 12 & Chloramphenicol & 37.2 \\
\hline 13 & Amoxicillin & 34.8 \\
\hline 14 & Cefoxitin & 32.5 \\
\hline 15 & Cefuroxime & 32.5 \\
\hline 16 & Azithromycin & 32.5 \\
\hline 17 & Cephalothin & 23.2 \\
\hline 18 & Cefixime & 23.2 \\
\hline 19 & Gentamycin & 18.6 \\
\hline 20 & Norfloxacin & 18.6 \\
\hline
\end{tabular}

Yousefi Mashou fetal study in 1999 showed that the most common organisms isolated from blood samples taken among 104 blood cultures positive were Pseudomonas (26.9\%), Klebsiella (25\%), Staphylococcus aureus (14.4\%), E. coli (13.5\%), Staphylococcus epidermid is (7.7\%), respectively [14]. The study took place in America in 2002 by Huang and colleagues were the most common bacteria isolated from blood cultures consisted of coagulase-negative Staphylococcus (42\%), Staphylococcus aureus (16.5\%), Enterococcus faecalis (8.3\%), E. coli (7.2\%) and Klebsiella pneumoniae (3.6\%) [15]. Mahtaet al study in 2005 showed the prevalence of bacteria in blood cultures were as follows: Pseudomonas (19.75\%), E. coli (15.17\%), Klebsiella pneumoniae (14.99\%), Staphylococcus aureus (13.86\%) and Salmonella (12.87\%) [16]. In another study, Staphylococcus aureus, Acinetobacter, Pseudomonas aeruginosa, Enterobacter and Klebsiella had have among the first to fifth place [17]. In another study, the most common organisms isolated from blood cultures were of Enterobacter, Staphylococcus aureus, Pseudomonas aeruginosa [18]. Regarding to these results, although type of bacteria in positive specimens are in the same groups, the percentage and arrangement of them are different.

Arrangement of antibiotic susceptibility of common bacteria in the blood culture is different in several studies. Evaluation of antibiotic resistance in bacteria in the present study represents the largest Cefixime antibiotic resistance (90\%), Amoxicillin (86.7\%), Cefoxitin (85.7\%), Cefuroxime (85.7\%) and penicillin (78.3\%) was. In a study, maximum resistance to bacteria was of $75 \%$ and $100 \%$ toward Ampicillin and Tetracycline, respectively [11]. In another study, maximum resistance was reported to Ampicillin, Kanamycin, Cefetriaxone, Ceftazidime, respectively: 100, 100, 100 and 80 percent [19]. In this investigation the most resistant to coagulase negative staphylococci was seen in Cefixime, Impipenem, Cefteriaxone and Oxacillin (100\%) which is similar to other studies. However in our study number of resistant antibiotic groups to coagulase negative staphylococci was more than other studies. Resistance to this group in Penicillin and Oxacillin has been reported in various studies 
Table 3. Antibiotic resistance in common bacterial groups in blood cultures performed.

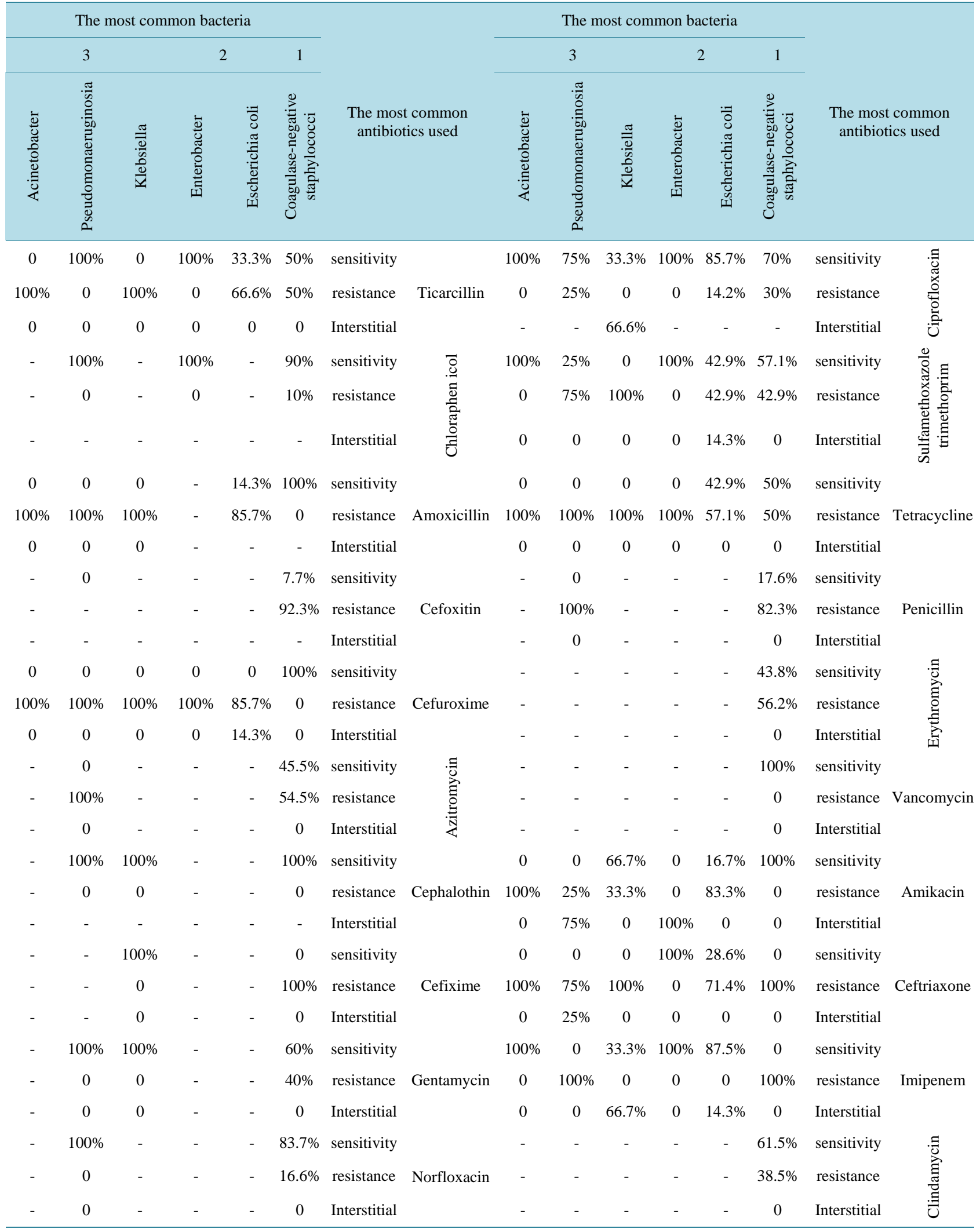

1-coagulase negative staphylococci.2-Enteric gram-negative bacteria 3. Non-fermentative Gram-negative bacilli. 
Table 4. Sensitivity and resistance to antibiotics.

\begin{tabular}{|c|c|c|c|}
\hline \multicolumn{2}{|c|}{ The rate of antibiotic resistance } & \multicolumn{2}{|c|}{ The rate of antibiotic sensitivity } \\
\hline Antibiotics & Percent of resistance & Antibiotics & Percent of sensitivity \\
\hline Cefixime & $90 \%$ & Chloramphenicol & $93.8 \%$ \\
\hline Amoxicillin & $86.7 \%$ & Vancomycin & $90 \%$ \\
\hline Cefoxitin & $85.7 \%$ & Ciprofloxacin & $75.6 \%$ \\
\hline Cefuroxime & $85.7 \%$ & Gentamycin & $75 \%$ \\
\hline Penicillin & $78.3 \%$ & Norfloxacin & $75 \%$ \\
\hline Ceftriaxone & $72.2 \%$ & Clindamycin & $64.7 \%$ \\
\hline Tetracycline & $65.4 \%$ & Imipenem & $52.8 \%$ \\
\hline Azithromycin & $64.3 \%$ & Erythromycin & $52.4 \%$ \\
\hline Ticarcillin & $52.9 \%$ & Ticarcillin & $47.1 \%$ \\
\hline Sulfamethoxazole-trimethoprim & $51.4 \%$ & Sulfamethoxazole-trimethoprim & $45.7 \%$ \\
\hline Erythromycin & $47.6 \%$ & Amikacin & $36.8 \%$ \\
\hline Amikacin & $42.1 \%$ & Azithromycin & $35.7 \%$ \\
\hline Clindamycin & $35.3 \%$ & Tetracycline & $34.6 \%$ \\
\hline Imipenem & $24.9 \%$ & Ceftriaxone & $22.4 \%$ \\
\hline Gentamycin & $25 \%$ & Penicillin & $17.4 \%$ \\
\hline Norfloxacin & $25 \%$ & Cefoxitin & $14.3 \%$ \\
\hline Ciprofloxacin & $19.5 \%$ & Amoxicillin & $13.3 \%$ \\
\hline Chloramphenicol & $6.3 \%$ & Cefoxitin & $10 \%$ \\
\hline Vancomycin & $5 \%$ & Cefuroxime & $7.1 \%$ \\
\hline
\end{tabular}

[11] [20]-[22]. It seems that the use of antibiotics in our study resulted in a lack of proper comparison with other studies in the family of bacteria. There was the greatest resistance to Amoxicillin, Cefuroxime and Amikacin and E. coli. In another study, the greatest resistance to E. coli than Sulfamethoxazole-Ttrimethoprim (28\%) and ciprofloxacin (3\%) had developed and there was no resistance to the Fluoroquinolones [23]. In another study, eftazidime, Gentamicin and Cotrimoxazole were most resistant to antibiotics than E. coli [24]. Klebsiella had $100 \%$ resistance rather than antibiotics Sulfamethoxazole-Trimethoprim, Tetracycline, Ceftriaxone, Tikarcillin, Amoxicillin and Cefuroxime. While in other studies the most resistance was to Ceftizoxim and Gentamicin against Klebsiella [24]. In another study, the greatest resistance to bacteria was referred to Ampicillin and Tetracycline [11]. Also Pseudomonas aeruginosa had 100\% resistance to Tetracycline, Penicillin, Imipenem, Azithromycin, Cefuroxime, Amoxicillin and Chloramphenicol. While in other studies it has had the most resistant to Gentamicin, Ceftazidime and Ceftizoxim [24]. In another study, most antibiotic-resistant to these bacteria was related to Ampicillin and Tetracycline [11]. In another study, this resistance to Ciprofloxacin, Ceftazidime and Imipenem was found [23]. These results are similar to the present study.

Despite similarities in the resistance pattern compared to other studies, in this study of Rasht, the pattern is special and unique model and based on the requirement of Guilan province's health. Considering the point that the experimental treatment and sometimes in appropriate antibiotics, also use of inappropriate doses are in effective types of bacteria becoming resistant to the antibiotics, even of new are effective. It is necessary to pay more attention to the results of the antibiogram of bacteria isolated from blood cultures. Also it is necessary to prevent over the counter shopping of antibiotics in pharmacy to people in regarding to pull up antibiotic resistance. Considering that the present study was a retrospective study and variables were not considered by authority of researchers. It is necessary to do large and continuous prospective study in Guilan province and optimized in- 
forming through labs, especially reference labs to doctors and hospitals should be exposed to all these phenomena with more knowledge to confront to consider these issues in their empiric treatments. Also it is recommended to use resistance pattern and susceptibility from the same patterns in reference laboratory to be more comprehensive. Also registration system and similar network of provincial between all of laboratories including public and private under the province deputy of hygienic can better inform all doctors to facilitate and it has been a remarkable help at similar information.

\section{Acknowledgements}

At the end we thanks from Razi laboratory management in Rasht city, all laboratory colleagues, especially Mr Doctor Mesbah who helped us in this research.

\section{References}

[1] Mahon, C.R., Manuselis, G. and Lehman, D.C. (2007) Text Book of Diagnostic Microbiology. 3th Edition, Saunders, USA, 321, 373, 383, 527.

[2] Michael, B. and Richard, P. (2010) Organization for Infection Control. In: Mandell, G.L., Bennett, J.E. and Dolin, R., Eds., Principles and Practice of Infectious Diseases, 7th Edition, Churchill Livingstone, Philadelphia, 3669-3676.

[3] Naderi, H.R., Bakhshaei, M., Ghazvini, K., Zamaniyan, A. and Haghighi, J. (2006) The Prevalence of Nasopharyngeal Carriers Moraksella Kataralys in Healthy Children under 6 Years in Kindergartens of Mashhad City and Determination Antibiotic Resistance Pattern of Isolated in Moraksellla Kataralys. Journal of the Ear, Throat, Nose and Throat Iran, 18, 169-173.

[4] Masomi, J., Yadegari, D. and Mozoni, Sh. (2005) More Appropriate Antimicrobial Agents for Antibiogram. Iranian Journal of Infectious Disease and Tropical Medicine, 10, 53-58.

[5] Longo, D.L, Kasper, D.L, Jameson, J.L, Fauci, A.S, Hauser, S.L. and Loscalzo, J. (2011) Harrison, Sepsis and Septic Shock: Principles of Internal Medicine. 18th Edition, McGraw-Hill co., Boston.

[6] Karimishahidi, M., Dabbagh Mohammadi, G.H., Mohajer Irvani, B. and Meigooie, A. (2002) Evaluation of Inpatient Patients' Blood Cultures of Common Microorganisms. Changes in the Epidemiology and Antibiogram Amir Alam Hospital, 79-1377. Journal of Medicine Tehran University of Medical Sciences, 60, 31-37.

[7] Pirzade, T. and Nahaei, M.R. (2002) Bacteria Isolated from Blood Cultures at the Educational and Therapeutic Center Imam Khomeini, Iran. Journal of Tabriz University of Medical Sciences, 56, 40-45.

[8] Spencer, R.C. (1991) Anaerobic Bacteremia. In: Duerden Brian, I. and Drasar, B.S., Eds., Anaerobic in Human Disease, Edward Arnold, England, 324-342.

[9] Forbes, B.A., Sahm, D.F. and Weissfeld, A. (2007) Bloodstream Infections. In: Baily \& Scotts Diagnostic Microbiology, 20th Edition, Mosby Company, USA, 778.

[10] Ghafoori, A., Eslami, Z. and Saffari, F. (2009) The Prevalence of Aerobic and Anaerobic Bacteremia in Patients with Cancer and Their Antibiotic Resistance Patterns. Journal of Infectious Diseases and Tropical Medicine, Infectious Diseases Specialist Association, 14, 19-26.

[11] Sedighian, F., Saneii, A., Allaodolehi, H., Arashi, M. and Rekabpoor, Kh. (2008) Resistance to isolated microorganisms in Yahyanejad hospital, Babol. Medical Laboratory Journal, 2, 29-35.

[12] Saderi, H., Karimi, A. and Looni, M. (2009) The Frequency of Bacteria Isolated from Blood Cultures and Their Antibiotic Susceptibility Patterns in a University Hospital in Tehran. Iranian South Medical Journal, 12, 142-148. (in Persian)

[13] Mamishi, S., Pourakbari, B., Ashtiani, M.H., et al. (2005) Frequency of Isolation and Antimicrobial Susceptibility of Bacteria Isolated from Blood Stream Infections at Children's Medical Center, Tehran, Iran, 1996-2000. International Journal of Antimicrobial Agents, 26, 373-379. http://dx.doi.org/10.1016/j.ijantimicag.2005.08.004

[14] Yosefi, M.R. (1999) Bacteriology of Neonatal Septicemia and Determination of Their Antibiotic Resistance in Hamadan in 1998 to 1999 (Persian). Iranian South Medical of Journal, 2, 136-143.

[15] Huang, S.S., Labus, B.J., Samuel, M.C., et al. (2002) Antibiotic Resistance Patterns of Bacterial Isolates from Blood in San Francisco County, California, 1996-1999. Emerging Infectious Diseases, 8, 195-201. http://dx.doi.org/10.3201/eid0802.010102

[16] Mehta, M., Dutta, P. and Gupta, V. (2005) Antimicrobial Susceptibility Pattern of Blood Isolates from a Teaching Hospital in North India. Japanese Journal of Infectious Diseases, 58, 174-176.

[17] Yinnon, A.M., Schlesinger, Y., Gabbay, D. and Rudensky, B. (1997) Analysis of 5 Years of Bacteraemias: Importance of Stratification of Microbial Susceptibilities by Source of Patients. Journal of Infection, 35, 17-23. 
http://dx.doi.org/10.1016/S0163-4453(97)90857-4

[18] Shehabi, A.A. and Baadran, I. (1996) Microbial Infection and Antibiotic Resistance Patterns among Jordanian Intensive Care Patients. Eastern Mediterranean Health Journal, 3, 515-520.

[19] Habibian, R., Imani, R. and Khoshdel, A. (2012) Changes of Trend of Antibiotic Susceptibility in Isolated Bacteria from Culture of ICU Patients of Shahrekord Ayatollah Kashanani Hospital, I.R. Iran. Journal of Shahrekord University of Medical Sciences, 14, 77-83.

[20] Aminzadeh, Z., Vahdani, P. and Khosravi, Z. (2005) Sensitivity and Resistance of Microorganisms in Loghman Hakim Hospital, Tehran, 1381. Iranian Journal of Infectious Disease and Tropical Medicine, 10, 47-52.

[21] Sader, H.S., Jones, R.N., Dowzicky, M.J. and Fritsche, T.R. (2005) Antimicrobial Activity of Tetracycline Tested against Nosocomial Bacterial Pathogens from Patients Hospitalized in the Intensive Care Unit. Diagnostic Microbiology and Infectious Disease, 52, 203-208. http://dx.doi.org/10.1016/j.diagmicrobio.2005.05.002

[22] Tavakoli, A., Yazdani, R. and Bokaeian, M. (2001) Relative Frequency Study of Coagulase Positive Staphylococci Resistance to Beta Lactam Antibiotics Using Iodometric and Acidometric Assays. Tabib-E-Shargh, 1, 1-7.

[23] Huang, S.S., Labus, J.B., Samuel, M.C., Wan, D.T. and Reingold, A.L. (2002) Antibiotic Resistance Patterns of Bacterial Isolates from Blood in San Francisco County, California, 1996-1999. Emerging Infectious Diseases, 8, 195-201. http://dx.doi.org/10.3201/eid0802.010102

[24] Mohamad, A.A., Yadegari, D., Hoseinimoghadam, M.M., Shadnoosh, M., Farahanchi, N., Rafiazade, R. and Daneshvar, S. (2007) Antibiotic Resistance Pattern of in Gram-Negative Microorganisms Nosocomial during the Years 2000-2004 in Anbiya Hospital Tehran. Researcher (Research Journal Martyr Beheshti University of Medical Sciences), 11, 123-127. 
Scientific Research Publishing (SCIRP) is one of the largest Open Access journal publishers. It is currently publishing more than 200 open access, online, peer-reviewed journals covering a wide range of academic disciplines. SCIRP serves the worldwide academic communities and contributes to the progress and application of science with its publication.

Other selected journals from SCIRP are listed as below. Submit your manuscript to us via either submit@scirp.org or Online Submission Portal.
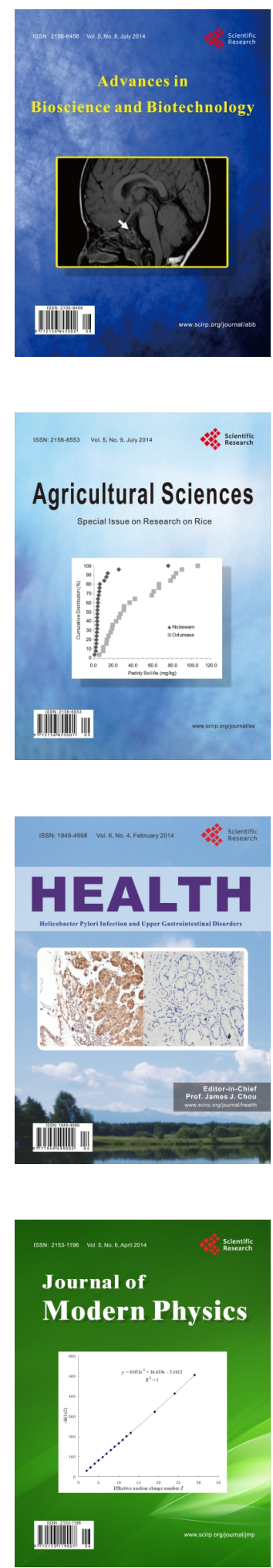
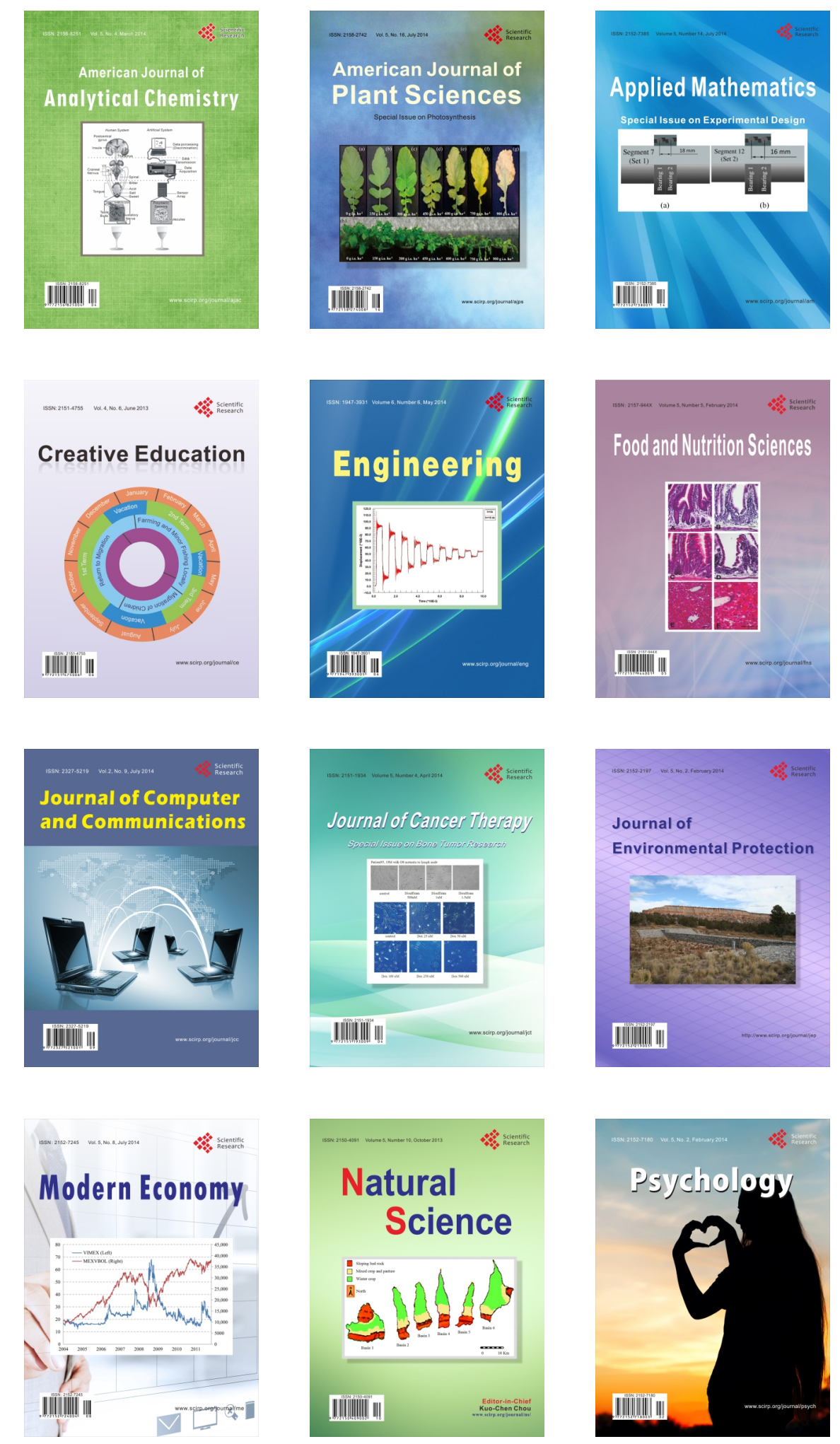